\title{
Development of a Methodology for Simulating Seat Back Interaction Using Realistic Body Contours
}

\author{
MAtTheW P. ReEd \\ JINGWEN HU
}

UniVERSITY OF Michigan TRANSPORTATION RESEARCH INSTITUTE 
Development of a Methodology for Simulating Seat Back Interaction Using Realistic Body Contours

\author{
Matthew P. Reed \\ Jingwen $\mathrm{Hu}$
}

The University of Michigan

Transportation Research Institute

Ann Arbor, Michigan 48109-2150

U.S.A.

Report No. UMTRI-2011-28

July 2011 
Technical Report Documentation Page

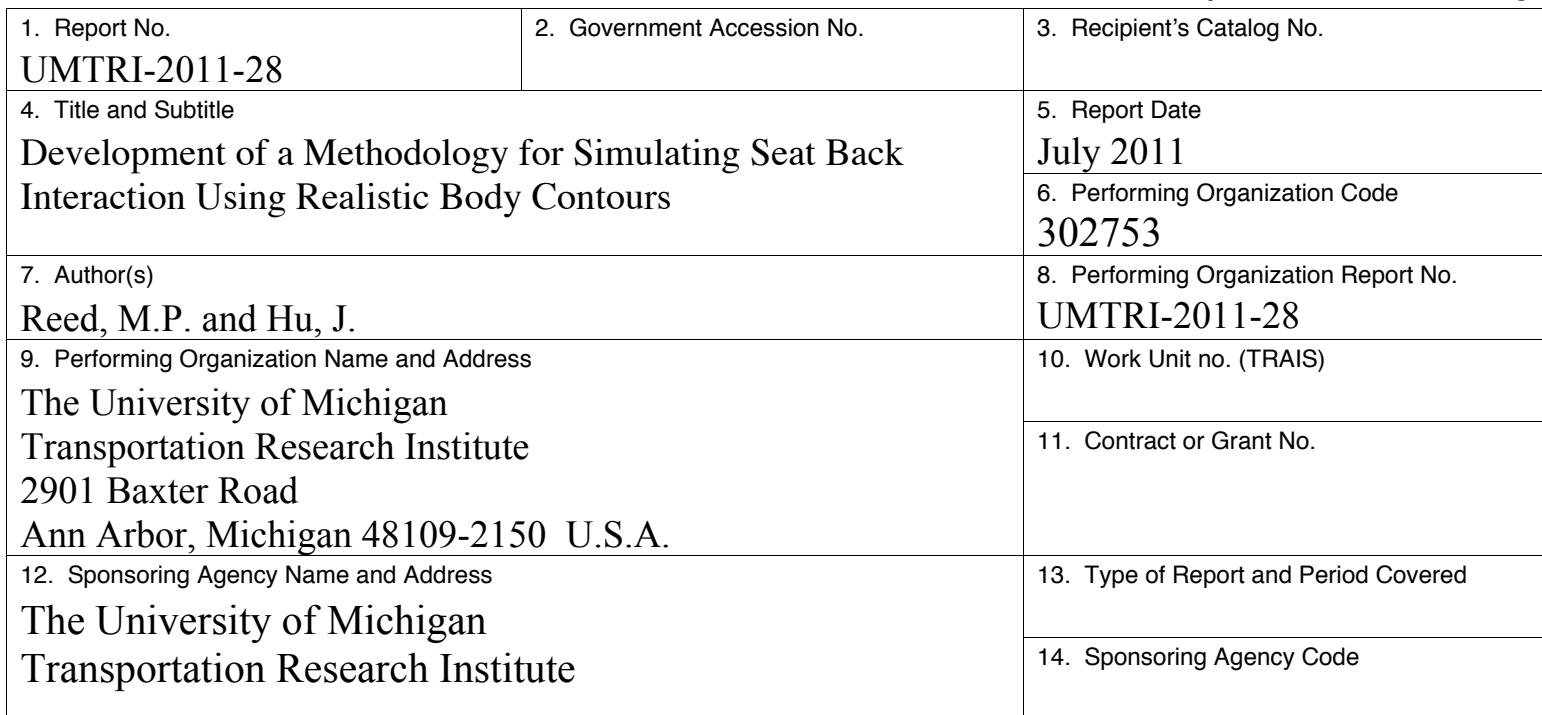

15. Supplementary Notes

16. Abstract

Seat comfort is driven in part by the fit between the sitter and seat. Traditional anthropometric data provide little information about the size and shape of the torso that can be used for backrest design. This report introduces a methodology for using three-dimensional computer models of the human torso based on a statistical analysis of body shapes for conducting automated fit assessments. Surface scan data from 296 men and 417 women in a seated posture were analyzed to create a body shape model that can be adjusted to a range of postures spanning those typical of vehicle occupants. A parameterized finite-element model of an auto seat surface was created, along with custom software that generates body models and postures them in the seat. A simple simulation technique was developed to rapidly assess the fit of the torso relative to the seat back. Further refinement of the method will allow prediction of seat surface pressure distribution, which may be usefully related to subjective assessment of seat fit.

17. Key Words

seat design, seat comfort, anthropometry, three-dimensional anthropometry

19. Security Classification (of this report)

20. Security Classification (of this page)

None

18. Distribution Statement

Unlimited

None

\begin{tabular}{|c|c|} 
21. No. of Pages & 22. Price \\
16 & \\
\hline
\end{tabular}




\section{ACKNOWLEDGMENTS}

This research was funded by Faurecia. The authors thank Samuel Baudu, Yang Cao, Michael Kellar, and Dana Lowell for their contributions to the project. 


\section{CONTENTS}

ACKNOWLEDGMENTS .................................................................................. iii

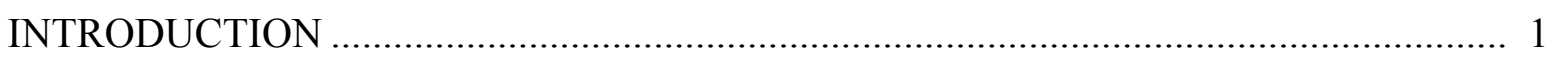

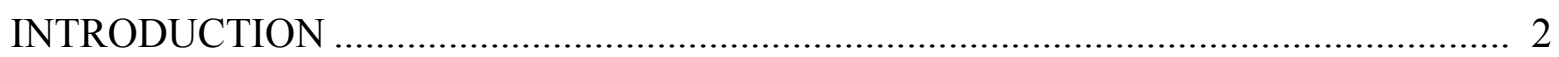

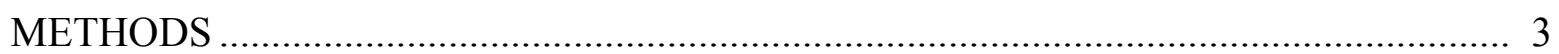

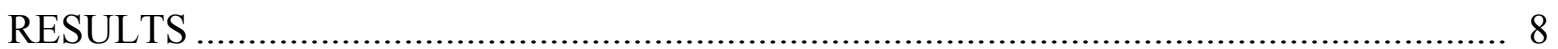

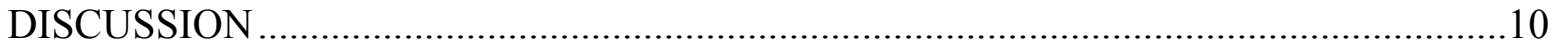

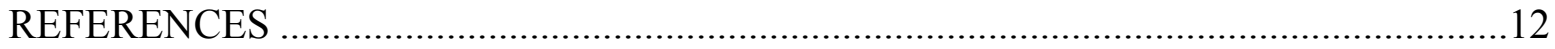




\begin{abstract}
Seat comfort is driven in part by the fit between the sitter and seat. Traditional anthropometric data provide little information about the size and shape of the torso that can be used for backrest design. This report introduces a methodology for using threedimensional computer models of the human torso based on a statistical analysis of body shapes for conducting automated fit assessments. Surface scan data from 296 men and 417 women in a seated posture were analyzed to create a body shape model that can be adjusted to a range of postures spanning those typical of vehicle occupants. A parameterized finite-element model of an auto seat surface was created, along with custom software that generates body models and postures them in the seat. A simple simulation technique was developed to rapidly assess the fit of the torso relative to the seat back. Further refinement of the method will allow prediction of seat surface pressure distribution, which may be usefully related to subjective assessment of seat fit.
\end{abstract}




\section{INTRODUCTION}

Dimensional mismatch between a seat and sitter can cause discomfort. For example, a backrest that is too narrow can create discomfort at the sides of the body and can also diminish the effectiveness of a lumbar support. Traditional anthropometric data (manually measured lengths, widths, circumferences) provide little guidance for seat back design because few dimensions are available that are closely related to seat design parameters. In particular, traditional anthropometric data do not provide guidance on contours.

In the past decade, surface-scanning equipment has revolutionized anthropometry by allowing rapid recording of whole-body surface shapes. The Civilian American and European Anthropometry Resource (CAESAR) project was the first large-scale study to scan U.S. civilians. In addition to standard anthropometric measures, scans were recorded in one standing and two seated postures for a convenience sample of 2400 U.S. adults from 18 to 65 years old. Body landmark locations were extracted from the scans.

The application of the CAESAR scan data for seat design has been hampered by difficulties in working with the data and the need to develop new methodologies to apply the data in design. Each set of raw scan data typically contain over 500,000 unstructured data point and represent a single posture. One approach to using the CAESAR data has been to manually craft manikins based on a small number of selected scans for use in human modeling software packages used for ergonomics, such as Jack and Ramsis. However, this method takes advantage of only a small fraction of the available information.

An alternative approach builds a statistical model of body shape using a large number of subjects. First introduced by Allen et al. (2003), the method has been applied specifically to ergonomic analysis of seating by Reed and Parkinson (2008). In the current work, the previously developed torso shape model was augmented to allow a range of seated postures to be represented. Each of the original seated body mesh models in the scanned posture was morphed to 15 alternative postures by manipulating two postural variables. A PCAR analysis on the results produced a model that predicts body shape as a function of recline, lumbar spine flexion, stature, and BMI for both men and women.

A generic finite-element model of a seat was created using shell elements. Landmark locations on the seat model are used to morph it to match any desired seat contour. An automated software procedure as developed to simulate the movement of the sitter, defined using the statistical body shape model, into the seat model. The resulting penetration was assessed with respect to seat fit. 


\section{METHODS}

\section{Posturable Torso Model}

Figure 1 shows the modeling methodology. Torso data from relaxed seated scans for 296 men and 417 women, including all of the obese subjects in the U.S. CAESAR sample, were extracted for analysis. Surface data for the arms and legs were removed to isolate the parts of the body that normally interact with seats. A uniform mesh was fit to the data, exploiting the fact that the data were sampled in horizontal slices (60 slices x 60 points per slice). A statistical analysis was then conducted on the vertices of the mesh, along with the associated body landmarks and internal joint locations estimated from the surface landmark locations. The analysis method (PCAR) uses principal component analysis to obtain a simpler representation of the data, followed by a regression analysis to predict the body shape from subject covariates such as stature and body weight (Reed and Parkinson 2008).

The previously developed statistical model of torso shape represented only the scanned posture, which is not typical of vehicle occupant postures. For the current study, the original data were reanalyzed using a novel methodology. Each subject's scan model (a set of 3600 vertices representing the surface and associated landmarks) was articulated to a set of postures relative to the scanned posture, defined by two variables.

The entire model (thighs through head) was tipped rearward from zero to 30 degrees. The lumbar spine was flexed, relative to the measurement posture, by -15 to 30 degrees, with the lumbar motion evenly distributed between the estimated T12/L1 and L5/S1 joint locations. In all postures, the head was maintained at the measured global orientation by flexion or extension of the cervical spine, with motion evenly distributed between the estimated $\mathrm{C} 7 / \mathrm{T} 1$ and L5/S1 joint locations.

Flexion of the lumbar and cervical spine areas was accomplished using a radial-basisfunction (RBF) morphing method (Bennick et al. 2007). A "box" of eight reference nodes was constructed around each of the head, thorax, and pelvis/thighs, as shown in Figure 1. These reference nodes were articulated with the underlying skeletal linkage to each new posture. The change in locations of the nodes relative to the original positions was used to construct a $3 \mathrm{D}$ vector interpolation function using multiquadric basis functions (Bennick et al. 2007). This morphing function was applied to all of the surface nodes and landmarks in the original model to obtain a repostured model. The resulting models (15 per subject, including the scan posture) were analyzed using the PCAR method. For prediction, three posture variables were included. Thigh angle is the mean thigh angle with respect to horizontal. Lumbar flexion is estimated as two times the angle between the estimated lumbar (L5/S1 to T12/L1) and thorax (T12/L1 to C7/T1) skeletal segments. (Pelvis angle could not be reliably estimated from the available landmarks, so lumbar flexion could not be calculated directly.) Torso recline is the angle with respect to vertical of a vector from the midpoint between the estimated hip locations to C7/T1. These variables were calculated for each of the morphed scan models and used as covariates in the regression analysis. Separate models were created for men and women. 


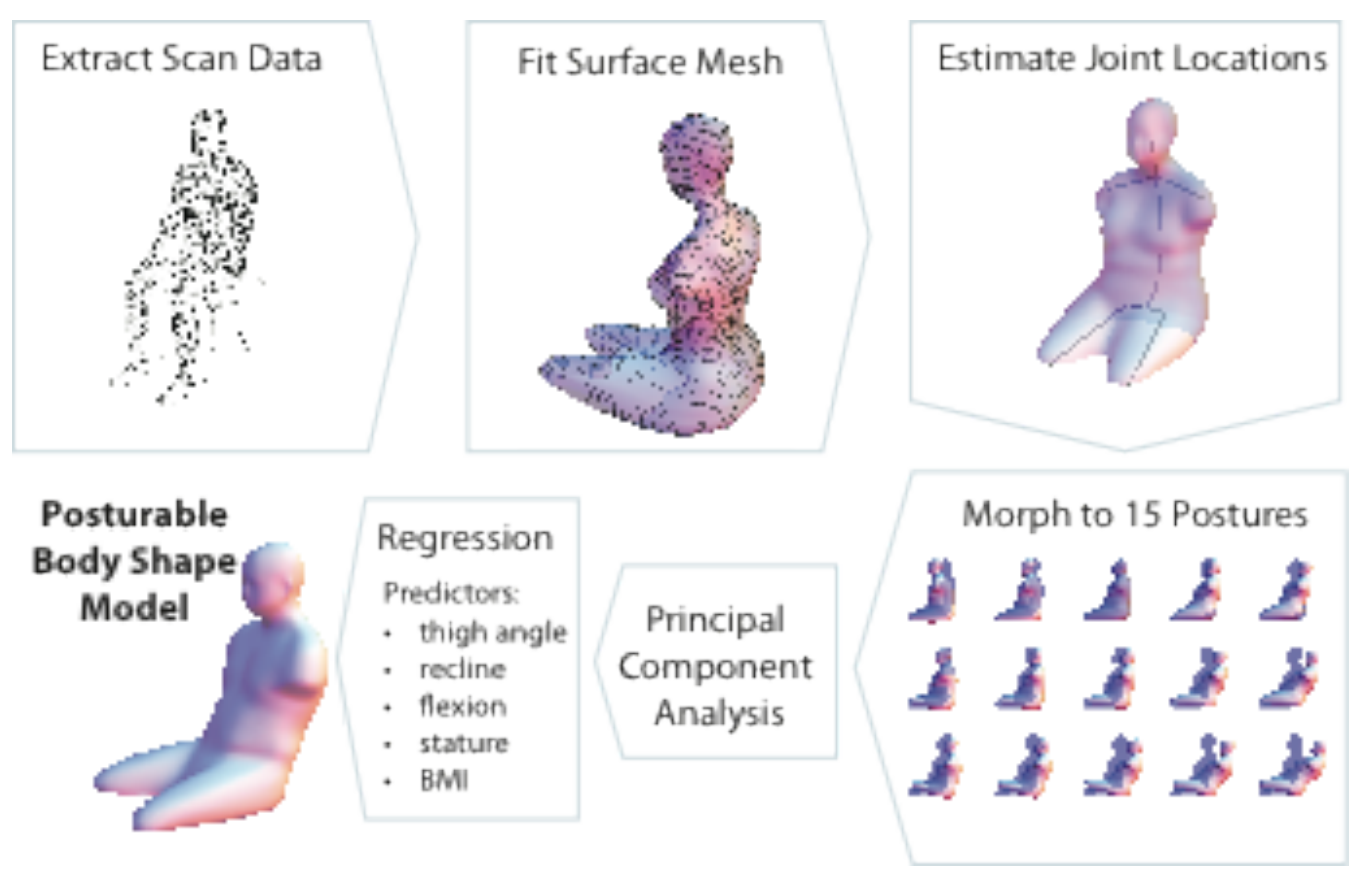

Figure 1. Schematic of process for creating a posturable body shape model from scan data.
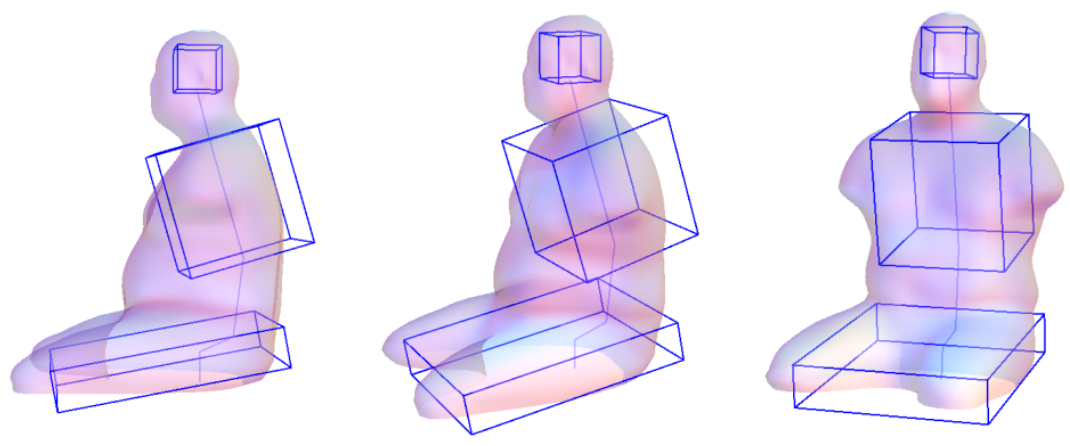

Figure 2. Head, thorax, and pelvis/thigh reference point boxes used to morph postures.
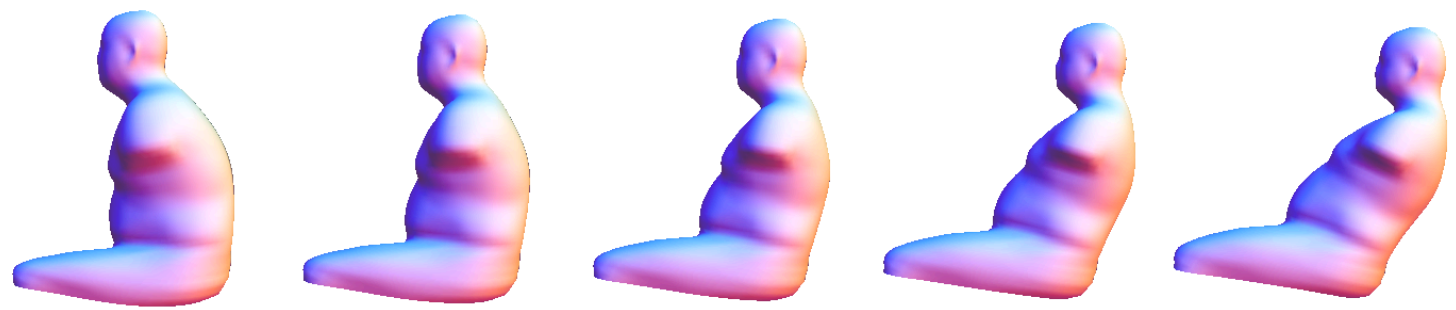

Figure 3. Result of exercising the male model over a range of postures with stature $=1755 \mathrm{~mm}$ and $\mathrm{BMI}=$ $40 \mathrm{~kg} / \mathrm{m}^{2}$. 


\section{Framework for Virtual Seat Fit Testing}

The goal of this effort is to develop a framework to conduct virtual fit assessments using a wide range of body sizes and shapes selected to represent the target user population. Each virtual fit will predict the seat surface deformation and pressure for a particular occupant, and statistical evaluation can be performed with multiple simulations for occupants with different body sizes and shapes.

Figure 4 shows the virtual fit process using finite element (FE) seat models and human models representing different body sizes and shapes. In this study, a program was written in Scilab, a free, open-source computing environment (www.scilab.org), to automatically generate the occupant FE model with specific body size, shape, and posture; integrate the seat FE model into the simulation; and setup the boundary conditions of virtual fit simulations.

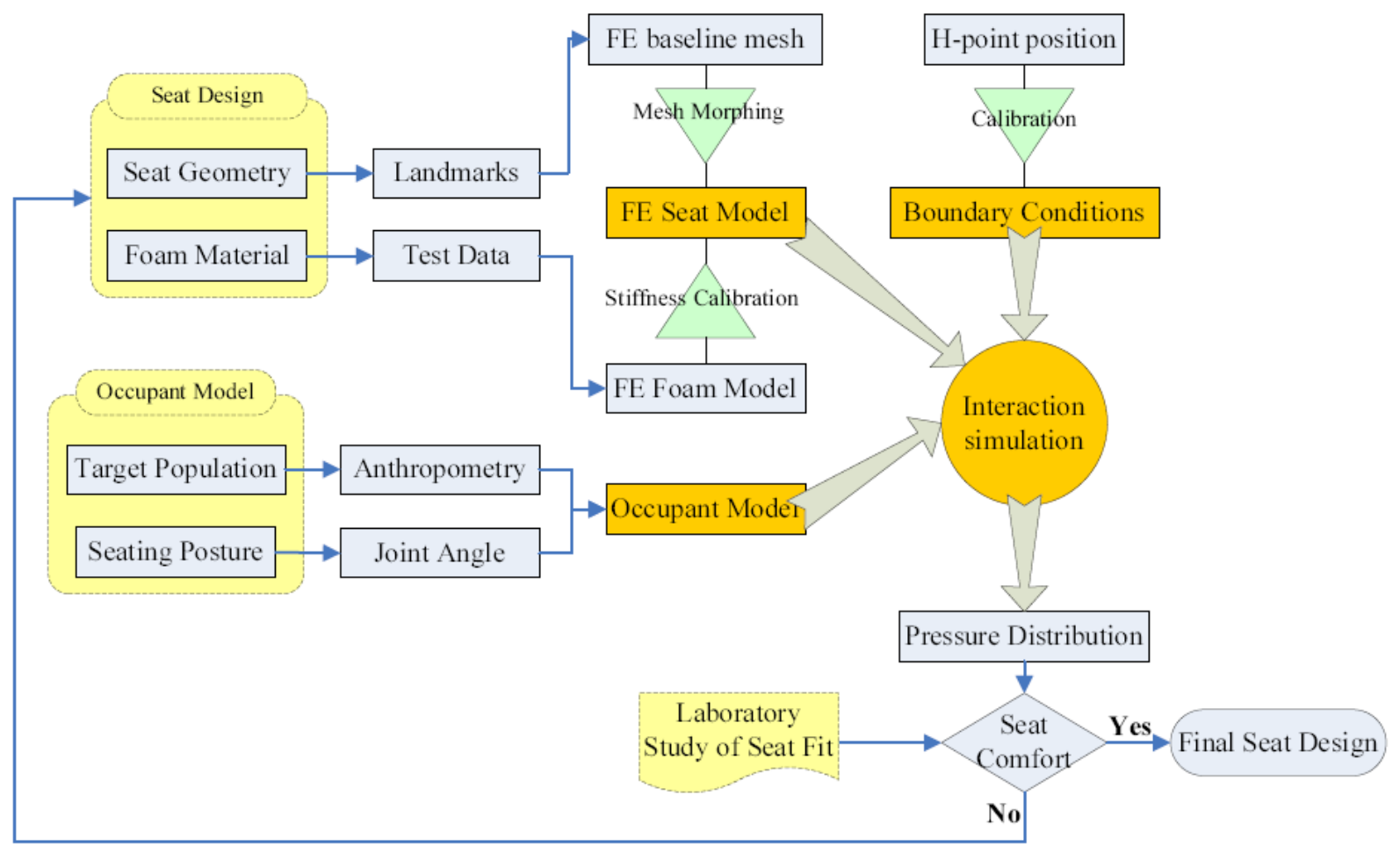

Figure 4. Flowchart of virtual fit methodology.

\section{Seat Model}

To shorten the calculation time, two simplified seat FE models were generated based on the surface geometry of two seat models. A layer of shell elements was used to represent the seat surface geometry. Two components, rigid base and elastic layer, were used for seat back as shown in Figure 5. The elastic layer was assigned a 3-mm thickness and an elastic material with $140 \mathrm{MPa}$ Young's modulus. Note that since the purpose of this study is to setup the framework and provide a demonstration of virtual fit, the stiffness of the seat model might not be very accurate. Further improvement can be made on the seat 
model, and a more complicated seat model can be easily implemented into the whole virtual fit process.

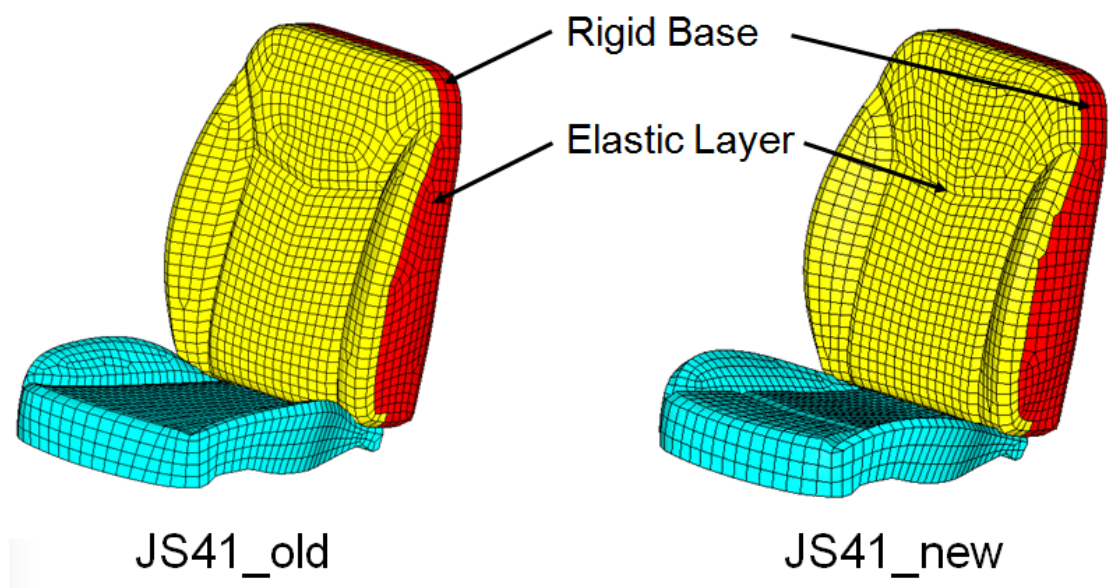

Figure 5. Simplified seat FE model with two seat geometries.

\section{Virtual Fit Simulation Setup}

Hypermesh 10.0 was used as the FE pre-processor to perform the meshing and generate a template, which was modified by the Scilab program to setup each simulation. LSDYNA 971 was used as the solver for all FE simulations. In the Scilab program, FE node coordinates for a group of occupants with different body sizes, shapes, and postures can be generated based on different sets of input parameters, including the height, BMI, thigh angle, lumbar flexion, and torso recline.

For each simulation, the setup is shown in Figure 6, in which the H-point of the occupant model was initially positioned $40 \mathrm{~mm}$ higher and $100 \mathrm{~mm}$ more forward compared to the seat H-point. Motion at about $0.1 \mathrm{~m} / \mathrm{s}$ was prescribed onto the occupant model, moving the occupant H-point (hip joint center location) toward the seat H-point. Surface-tosurface contact with friction coefficient of 0.1 was defined between the occupant model and the elastic layer of the seat back model. The rigid base of the seat back model was fixed throughout the simulation. By using the Scilab program, a group of simulations with different body sizes, shapes, and postures can be set up automatically and copied into different folders. An executive file can also be created to run all the simulations continuously. 


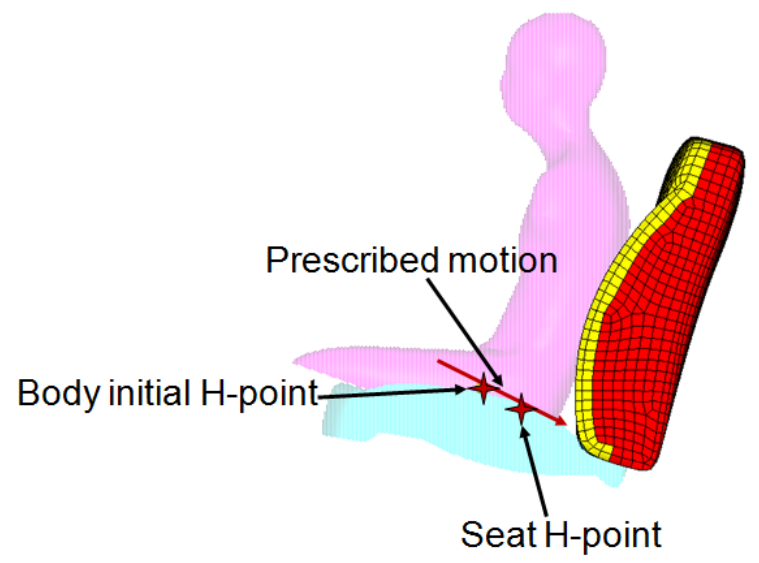

Figure 6. Virtual fit simulation schematic.

\section{Parametric Study}

To demonstrate the feasibility of this virtual fit program, a parametric study of 24 simulations was conducted. The parameters controlled in the parametric study are shown in Table 1. The seat back deformations on the elastic layer were output from each of 24 simulations. Each simulation took less than one minute.

Table 1.

Parameters for Parametric Study

Parameters

Stature

BMI

Recline

Lumbar spine flexion angle

Seat model
Levels

$165 \mathrm{~cm}, 175 \mathrm{~cm}$, and $185 \mathrm{~cm}$

20 and 35

Same as the cushion angle

5 and $15 \mathrm{deg}$

JS41_old and JS41_new 


\section{RESULTS}

Figures 7, 8, and 9 show some of the simulation results. Figure 7 shows that the new seat provides a different deformation distribution with the range of body sizes investigated.

Figure 8 indicates that higher lumbar spine flexion angle reduces the seat back deformation under the prescribed loading regime. Figure 9 demonstrates that the occupant with a higher BMI generates higher deformation on the seat back for this loading scenario. The results from the parametric study demonstrated that the virtual fit process developed in this study is sensitive to body size and shape.
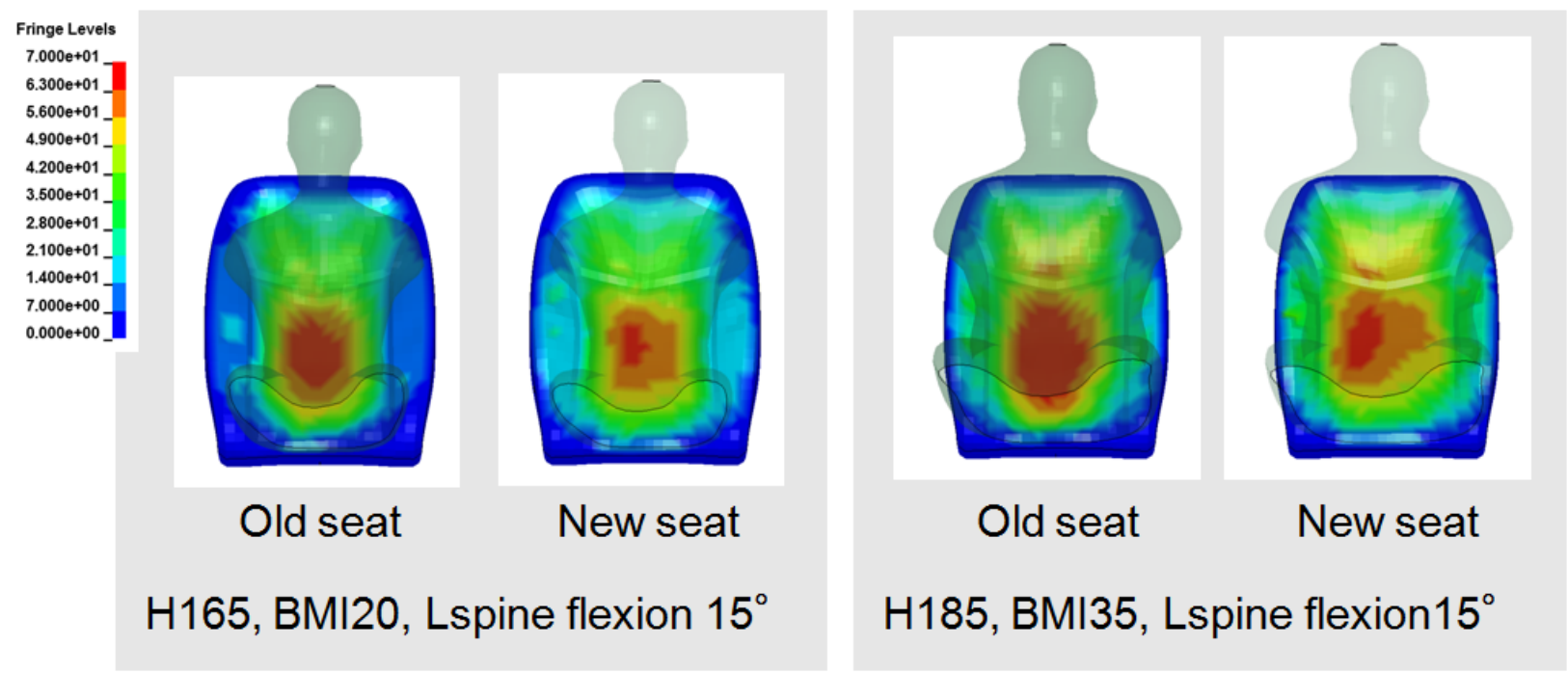

Figure 7. Simulation results for old seat vs. new seat
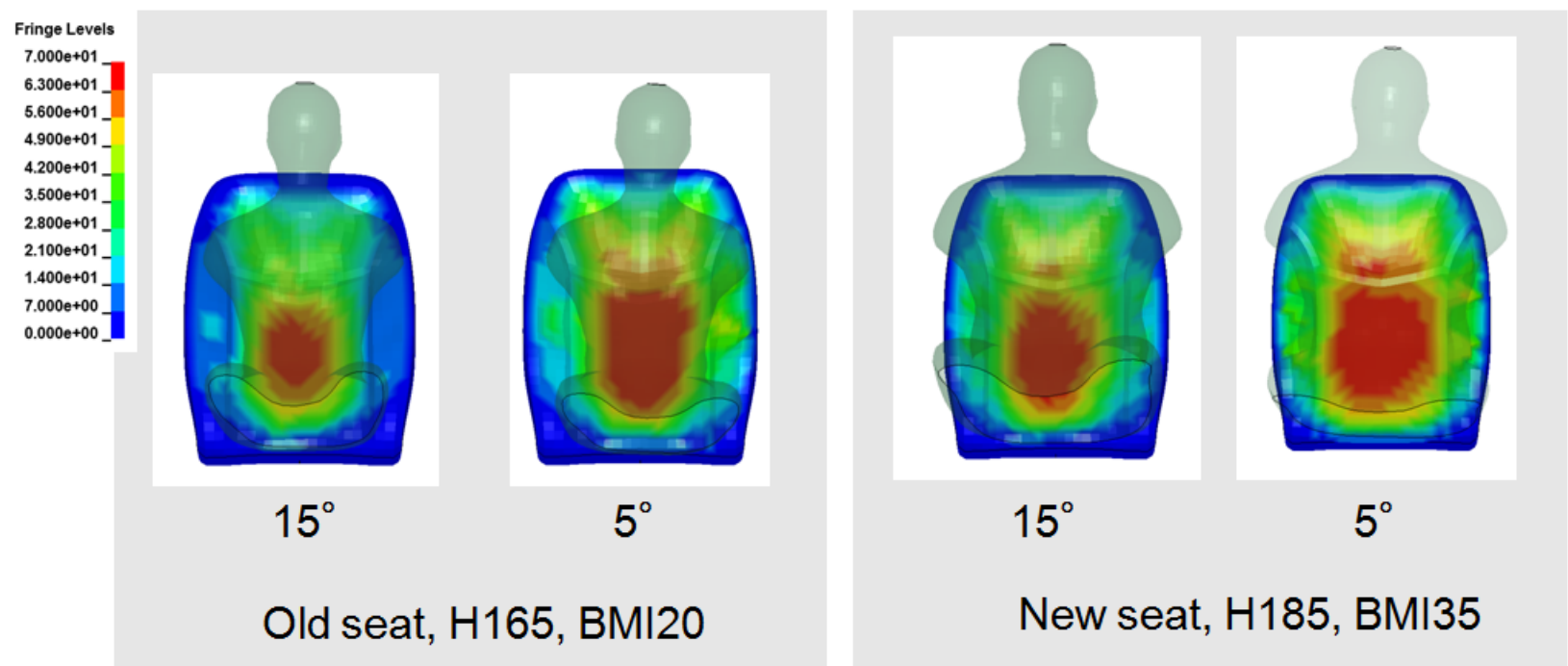

Figure 8. Simulation results for different lumbar spine flexion angles 


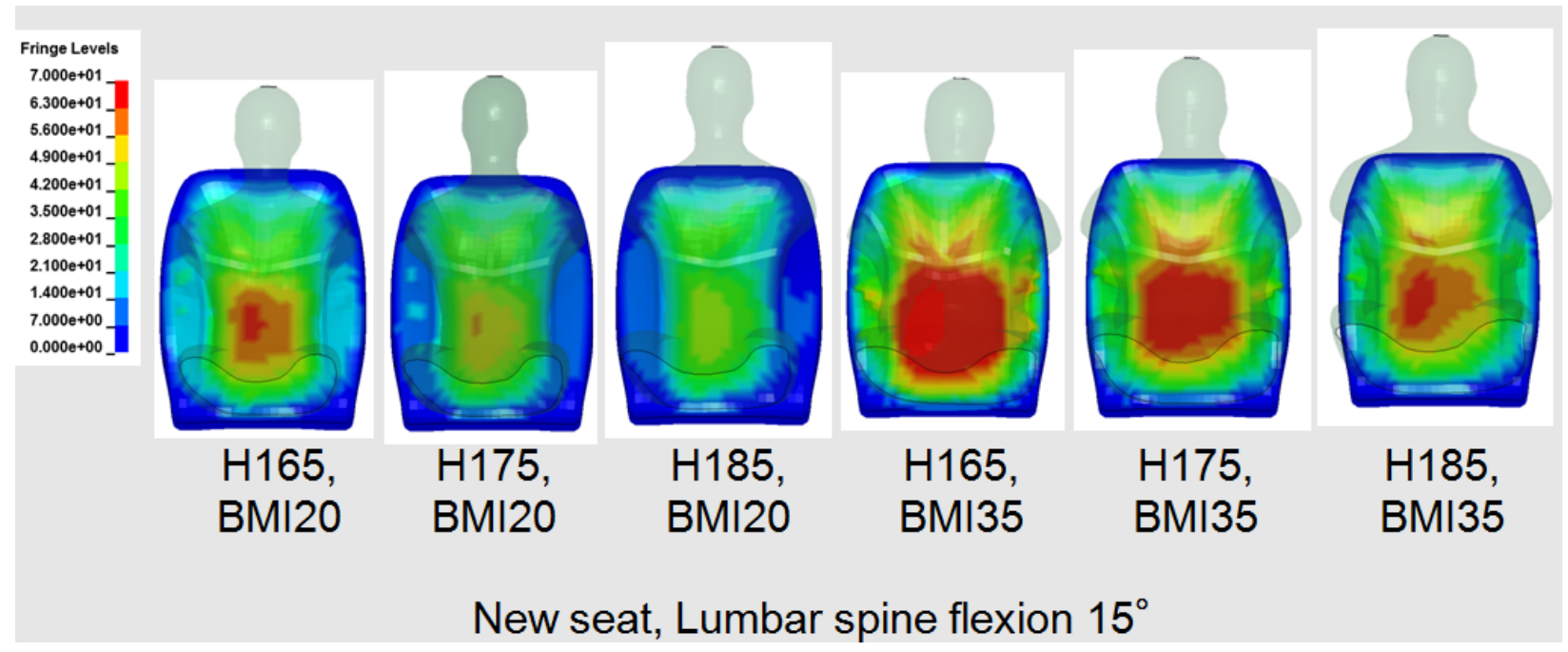

Figure 9. Simulation results for different torso shapes 


\section{DISCUSSION}

\section{Accomplishments}

This project demonstrated the feasibility of conducting virtual fit trials using a parametric human figure model and a simplified finite-element simulation of seatback loading. The work had several innovative elements:

- A method was developed and implemented to include posturing capability in a statistical model of torso shape.

- A framework was developed for rapidly morphing a seat model to match a target geometry based on a small set of landmarks.

- Automated methods for rapidly assembling a simulation with a large number of human body model and a seat back model were developed using an open-source software system.

- A simple prescriptive method for defining seatback interaction showed sensitivity for differentiating seat fit among seats and body shapes.

\section{Limitations}

This pilot work is limited by the small number of simulations performed. In particular, only two seats were examined for a small number of body shapes and postures, since the goal was to establish the feasibility of the method rather than to make judgments about the seats. The human body model is based on a fairly small sample (296 men and 417 women). Using a larger number of subjects (a total of 2400 are available in the U.S. CAESAR sample) could improve the fidelity of the body models. The posturing methodology is as yet unvalidated, both with respect to the body shape morphing and the effects of the seat on the posture; work ongoing at UMTRI will provide validation for this approach.

The most important limitation of the current work is that the simulated pattern of deflection on the seatback, which is the primary outcome measure, has not been validated. Data are needed the link the posture and body shape of sitters with a wide range of body shapes to physical measurements of seat back interaction. Moreover, the ultimate success of the method is dependent on the development of a quantitative, reliable method to predict subjective responses from the (predicted) physical interaction between the sitter and seatback.

\section{Next Steps}

The current work was envisioned as the first phase in a multi-stage research program leading to a fully developed virtual seat evaluation methodology. Three tasks are suggested for the next phase of the research: 
1. Improve the human body shape model by adding more subjects and both improving and validating the posturing functionality.

2. Conduct a laboratory study with human volunteers to quantify the relationships between subjective fit and objective measures of seatback interaction, in the process generating data that can be used to validate simulations.

3. Develop a validated FE method for simulating seatback interaction based on the framework developed in the current study. 


\section{REFERENCES}

Allen, B., Curless, B., and Popovic, Z. (2003). The space of human body shapes: reconstruction and parameterization from range scans. Proceedings of the 2003 International Conference on Computer Graphics and Interactive Techniques (SIGGRAPH). San Diego, CA.

Reed, M.P. and Parkinson, M.B. (2008). Modeling variability in torso shape for chair and seat design. DETC2008-49483. Proceedings of the ASME Design Engineering Technical Conferences. ASME, New York.

Bennink, H.E., Korbeeck, J.M., Janssen, B.J., Romenij, B.M. (2006). Warping a neuroanatomy atlas on 3D MRI data with radial basis functions. Proceedings of the 2006 International Conference on Biomedical Engineering. pp. 214-218. 